\section{RMD Open}

Rheumatic \&

Musculoskeletal Diseases

\title{
Thickness of the bone-cartilage unit in relation to osteoarthritis severity in the human hip joint
}

Louise Brøndt Hartlev, ${ }^{1}$ Rasmus Klose-Jensen, ${ }^{1}$ Jesper Skovhus Thomsen, ${ }^{2}$ Jens Randel Nyengaard, ${ }^{3}$ Lene Warner Thorup Boel, ${ }^{4}$ Mogens Berg Laursen, ${ }^{5}$ Trine Bay Laurberg, ${ }^{1}$ Andreas Wiggers Nielsen, ${ }^{1}$ Kristian Steengaard-Pedersen, ${ }^{1}$ Ellen-Margrethe Hauge ${ }^{1,6}$

To cite: Hartlev LB, Klose-Jensen R, Thomsen JS, et al. Thickness of the bonecartilage unit in relation to osteoarthritis severity in the human hip joint. RMD Open 2018;4:e000747. doi:10.1136/ rmdopen-2018-000747

- Prepublication history and additional material for this paper are available online. To view these files, please visit the journal online (http://dx.doi. org/10.1136/rmdopen-2018000747)

Received 19 June 2018 Revised 7 August 2018 Accepted 9 August 2018

\section{Check for updates}

(c) Author(s) (or their employer(s)) 2018. Re-use permitted under CC BY. Published by BMJ.

For numbered affiliations see end of article.

Correspondence to Professor Ellen-Margrethe Hauge; ellhau@rm.dk

\section{ABSTRACT}

Objective Bone formation is a hallmark of osteoarthritis $(0 A)$. It has been speculated that bone formation may occur because of ossification at the bone-cartilage unit, that is, bone formation directly involving the calcified cartilage (CC). This study aimed to investigate the thickness of the $\mathrm{CC}$ and subchondral bone (SCB) in relation to the severity of the overlying articular cartilage (AC) degeneration.

Design We investigated femoral heads from 20 patients with $\mathrm{OA}$ and 15 healthy subjects with design-based stereology using systematic uniform random sampling of the entire joint surface. This was combined with the Osteoarthritis Research Society International (OARSI) OA cartilage histopathology assessment system, thus obtaining focal OARSI grades paired with thickness measurements of AC, CC and the SCB.

Results The patients with OA had thicker CC (mean 159; $95 \% \mathrm{Cl} 144$ to $177 \mu \mathrm{m}$ ) compared with the healthy subjects (mean 132; 95\% Cl 113 to $1550 \mu \mathrm{m} ; \mathrm{p}=0.036$ ), and this difference was even higher in areas without loss of AC thickness (OARSI grade $\leq 3) ; 187(95 \% \mathrm{Cl} 164$ to 214) $\mu \mathrm{m}$ vs 132 (95\% Cl 113 to 155$) \mu \mathrm{m}(\mathrm{p}=0.001)$. In the patients with $\mathrm{OA}$, a thicker SCB was observed in areas with loss of $A C$ thickness (OARSI grade $\geq 4$ ), but not in areas without loss of AC thickness (OARSI grade $\leq 3$ ).

Conclusion The study showed that thicker CC is present in early stages of $O A$, suggesting that bone formation by endochondral ossification is an early phenomenon of OA. Thickening of the SCB was present, but only in areas with denuded bone. Suggesting that also appositional bone growth occurs and that it may be a consequence of changed biomechanics.

\section{INTRODUCTION}

Hip osteoarthritis (OA) is not merely a disease of 'wear and tear' of the articular cartilage (AC). ${ }^{1}$ Bone formation is a hallmark of OA and may be part of the pathophysiology. ${ }^{2}$ Most studies of OA have focused separately on the $\mathrm{AC},{ }^{3}$ the subchondral bone $(\mathrm{SCB})^{2}$ or other joint tissues. ${ }^{4}$ Few studies have investigated

\section{Key messages}

What is already known about this subject?

- Alterations in the composition, functional properties and structure of the bone-cartilage unit occur during the development of osteoarthritis $(\mathrm{OA})$.

What does this study add?

- Calcified cartilage thickening is found, where no loss of articular cartilage has occurred, suggesting endochondral ossification to be an early phenomenon in $0 A$.

- Subchondral bone thickening is strongly associated with loss of articular cartilage and appears to be a late-stage phenomenon caused by appositional growth.

How might this impact on clinical practice?

- Possible disease-modifying interventions focused at the bone-cartilage unit might target different processes relevant for early or late $\mathrm{OA}$ disease stages.

the association between the tissues and their correlation with the degree of focal cartilage loss. ${ }^{56}$

It has been suggested that the thin layer of calcified cartilage (CC) between the AC and the SCB plate plays a role in the communication between the bone and cartilage in patients with $\mathrm{OA}^{7}$ Moreover, it has been suggested that the CC may be involved in endochondral ossification leading to bone formation in OA. ${ }^{8-10}$ Bone growth in OA has been confirmed by several studies, which found that the thickness of the SCB plate increased,,$^{511-15}$ in addition to the formation of osteophytes in OA. ${ }^{16-18}$ It remains, however, to be shown whether the growth of the SCB plate can be associated with either bone apposition or endochondral ossification. If bone growth occurs as a consequence of endochondral ossification, as have been 
Table 1 Characteristics of healthy subjects and patients with osteoarthritis

\begin{tabular}{|c|c|c|}
\hline & Healthy subjects $(n=15)$ & Patients with osteoarthritis $(n=20)$ \\
\hline Women:men (n) & $7: 8$ & 10:10 \\
\hline Age (years) & 63.6 (58.9 to 68.3$)$ & 64.3 (60.5 to 68.0 ) \\
\hline Weight $(\mathrm{kg})$ & 80.6 (66.4 to 94.8$)$ & 79.8 (73.2 to 86.5$)$ \\
\hline Height (cm) & 173.7 (168.0 to 177.3$)$ & 170.2 (166.0 to 174.0$)$ \\
\hline $\operatorname{BMI}\left(\mathrm{kg} / \mathrm{m}^{2}\right)$ & 26.8 (22.8 to 30.8$)$ & $27.5(25.5$ to 29.5$)$ \\
\hline Kellgren-Lawrence grade & - & 3.9 (3.8 to 4.0$)$ \\
\hline \multicolumn{3}{|l|}{ WOMAC score } \\
\hline Pain & - & 49.9 (39.4 to 60.3$)$ \\
\hline Stiffness & - & 58.2 (45.2 to 71.2 ) \\
\hline Physical activity & - & 49.9 (33.2 to 47.1$)$ \\
\hline
\end{tabular}

Data are presented as mean and $(95 \% \mathrm{Cl})$.

BMI, body mass index;WOMAC, Western Ontario and McMaster Universities Arthritis Index.

suggested, ${ }^{19}{ }^{20}$ then the tidemark would advance into the AC leading to a thickening of the $\mathrm{CC}$ and thinning of the AC. In contrast, bone apposition at the lower aspect of the SCB plate by modelling does not involve the CG. ${ }^{21}$ Therefore, a characterisation of the CC is essential in order to understand how the thickness of the SCB plate occurs during development and progression of OA.

Currently, it is difficult to investigate the CC in human $\mathrm{OA}$, since the $\mathrm{CC}$ cannot be identified in vivo by either conventional X-ray, CT or MRI. The CC, therefore, needs to be investigated using other methods, for example, histology.

The study aimed to investigate the thickness of the $\mathrm{SCB}$ and the CC and their relation to the different severity of AC damage in entire femoral heads from both patients with $\mathrm{OA}$ and healthy subjects. AC damage was determined by morphometric assessment of grade and stage (cf Osteoarthritis Research Society International (OARSI) scoring method) ${ }^{22}$ We hypothesise that the CC and SCB plate thickness increase progressively with cartilage damage in patients with $\mathrm{OA}$.

\section{MATERIALS AND METHODS Study population}

Human femoral heads from 35 subjects comprising 20 patients with OA with a mean age of 63.6 years (range $58.9-68.3$ years) and 15 healthy subjects with a mean age of 64.3 years (range 60.5-68.0 years) (table 1) were included in the study. ${ }^{5}$

Twenty femoral heads were obtained from patients with primary hip OA, who underwent hip replacement surgery at the Department of Orthopaedics at Farsoe Hospital in Denmark. The patients met the combined clinical and radiographic criteria of the American College of Rheumatology for OA. ${ }^{16}$ Patients with known bone metabolic diseases, secondary OA or other joint diseases, diabetes mellitus or malignant diseases were excluded from the study.
The femoral heads from the control group were obtained at autopsy from healthy subjects with macroscopically normal femoral heads. All healthy subjects had died suddenly from accidents or acute diseases. Subjects with a history of high-energy pelvic trauma or any signs of hip OA after macroscopic inspection were excluded. Furthermore, healthy subjects were excluded if they had any known diagnosis of bone metabolic disease, other joint diseases, diabetes mellitus or malignant diseases.

\section{Processing of tissue}

Immediately after removal of the femoral heads, they were fixated in $70 \%$ ethanol and processed according to a design-based stereological sampling scheme as previously described in detail. ${ }^{23}$ In brief: the entire femoral heads were rotated around a vertical axis (VA), which was perpendicular to the anatomical top of the femoral head (figure 1A). After choosing a random starting point, the femoral head was sawed using a diamond precision-parallel saw (Exakt Apparatebau, Norderstedt, Germany) into $7 \mathrm{~mm}$ thick parallel slices (figure 1B), which were halved, and alternating left and right half slices were randomly selected for the following microscopic evaluation ${ }^{23} 24$ (figure 1C). Depending on the size of the femoral head, a total of five to seven $7 \mathrm{~mm}$ thick halved parallel slices were collected from both patients with OA and healthy subjects. Each of the five to seven $7 \mathrm{~mm}$ thick halved parallel slices was embedded undecalcified in methylmethacrylate and cut into $7 \mu \mathrm{m}$ thick histological sections using a Jung model $\mathrm{K}$ microtome (R Jung, Heidelberg, Germany) equipped with a tungsten microtome knife. The sections were mounted and stained with May-Grünwald toluidine blue as previously described $^{23}$ (figure 1D).

\section{Microscopy and sampling}

The entire femoral head surface area was estimated by systematic uniform random sampling ${ }^{25}$ and vertical uniform random sections (figure 1). ${ }^{24}$ For each of the 

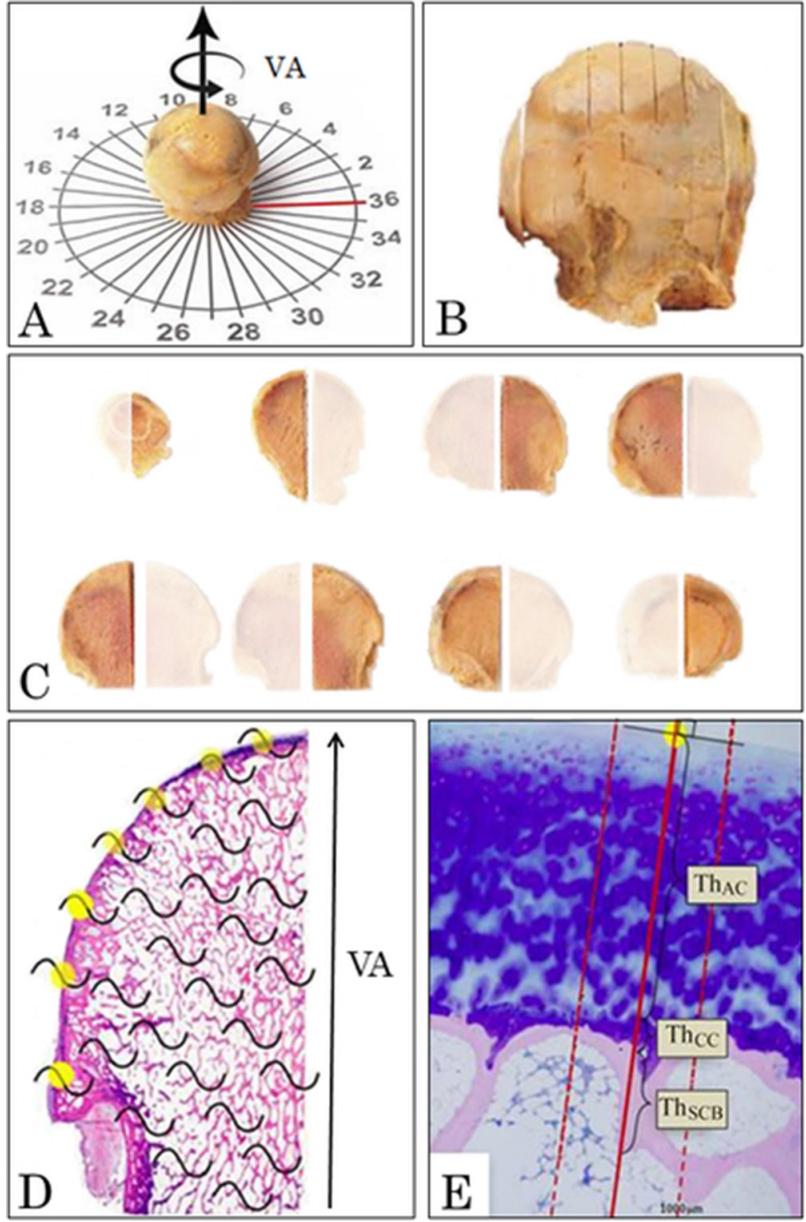

Figure 1 Design-based stereological methods using systematic uniform random sampling and vertical sections were used to study the undecalcified human femoral heads. (A) The vertical arrow represents the vertical axis (VA) of the femoral head and the rotated arrow represents the random rotation of the femoral head around the VA. The red line indicates the location of the medial part of the femoral head. (B) The femoral head was cut in $7 \mathrm{~mm}$ thick slices in a random orientation and parallel to the VA to get vertical uniform random sections. (C) The $7 \mathrm{~mm}$ thick slices were halved and alternating left and right half slices were sampled randomly resulting in a sampling fraction of one-half. (D) From each femoral head, all the sampled $7 \mathrm{~mm}$ thick halved parallel slices were embedded in methylmethacrylate, and 7 $\mu \mathrm{m}$ thick histological sections were cut from each slice and stained with May-Grünwald toluidine blue. In all histological sections, the joint surface was sampled systematically uniform random as every intersection (yellow dots) between the superimposed cycloid grids (black curved lines) and the joint surface. (E) For each random sampling point, the Osteoarthritis Research Society International (OARSI) grade was determined, and a line was drawn perpendicular to the joint surface. Along the line drawn perpendicular to the joint surface, the $\mathrm{Th}_{\mathrm{AC}}, \mathrm{Th}_{\mathrm{CC}}$ and $\mathrm{Th}_{\mathrm{SCB}}$ were measured. To increase the precision of the $\mathrm{Th}_{\mathrm{SCB}}$, two lines separated by $600 \mu \mathrm{m}$ were drawn parallel to and on each side of the line perpendicular to the joint surface. The subchondral bone (SCB) plate thickness was also measured along these additional two lines, and the average was used as the estimate of the SCB plate thickness at that specific sampling point. AC, articular cartilage; $\mathrm{CC}$, calcified cartilage. femoral heads, a total of 50-70 random sampling points were generated by superimposing a cycloid line grid (area per length, $\mathrm{a}(l)=2 \mathrm{~mm}$ ) onto each of the five to seven $7 \mu \mathrm{m}$ thick sections. The random sampling points were the intersections between the joint surface and the test lines of the cycloid line grid (figure 1D) ${ }^{26}$ For each random sampling point, the OARSI grade was determined using the OARSI scoring method described by Pritzker et $a l^{22}$ From all random sampling points, a line was drawn perpendicular to the joint surface, and along this line, the thickness of the AC, CC and SCB plate was measured (figure 1E). In order to increase the precision of the SCB plate thickness estimates, a parallel line was drawn on either side of the line perpendicular to the joint surface (figure 1E). These two lines were both separated by $300 \mu \mathrm{m}$ from the perpendicular line. For each random sampling point, the SCB plate thickness was measured along these three lines, and the average was used (figure 1E). Fovea capitis and osteophytes were excluded from the analysis. All measurements were performed by one observer, who was blinded for the group distribution.

The sections were evaluated under a light microscope (Olympus BH-2, Tokyo, Japan), equipped with a projection arm and a digitiser tablet (Wacom Intuos-3, Wacom, Otone, Japan) attached to a PC with SigmaScan Pro-5 (Systat Software, San Jose, CA) and a computer mouse mounted with a point-like red LED. The sections were analysed at a magnification of $\times 31$.

\section{OARSI grading, staging and scoring}

The OARSI grade (range 0-6) was determined according to the guidelines by Pritzker et al, where grading is based on structural and vertical changes of the joint surface which includes normal cartilage (grade 0), cartilage fibrillation (grade 1), discontinuity of the cartilage surface (grade 2), fissuring (grade 3), erosion and loss of cartilage thickness (grade 4), denudation of the surface (grade 5) and lastly deformation of the bone (grade $6){ }^{22}$ The OARSI stage (range 0-4) describes the horizontal extent of the articular joint surface irrespective of the underlying OARSI grade. ${ }^{22}$ The OARSI score was determined according to the recommended OA scoring method proposed by Pritzker et al. The OARSI score has a range of $0-24$ based on the most advanced grade and most extensive stage present. ${ }^{22}$

\section{Thickness measurements}

Corresponding measurements of the OARSI grade, the thickness of the AC $\left(\mathrm{Th}_{\mathrm{AC}}\right)$, the $\mathrm{CC}\left(\mathrm{Th}_{\mathrm{CC}}\right)$ and the $\mathrm{SCB}\left(\mathrm{Th}_{\mathrm{SCB}}\right)$ were made for each of the 50-70 random sampling points over the entire joint surface of each femoral head (figure 1).

The $\mathrm{Th}_{\mathrm{AC}}$ was measured as the orthogonal distance from the surface of the AC to the first encounter of the tidemark, while the $\mathrm{Th}_{\mathrm{CC}}$ was measured from the proximal tidemark to the cement line (figure $1 \mathrm{C}$ ). The term SCB is ambiguous. ${ }^{27}$ In this study, the SCB plate was 
Table 2 Comparison of OARSI and thickness measurements of articular cartilage, subchondral bone plate and calcified cartilage between healthy subjects and patients with osteoarthritis

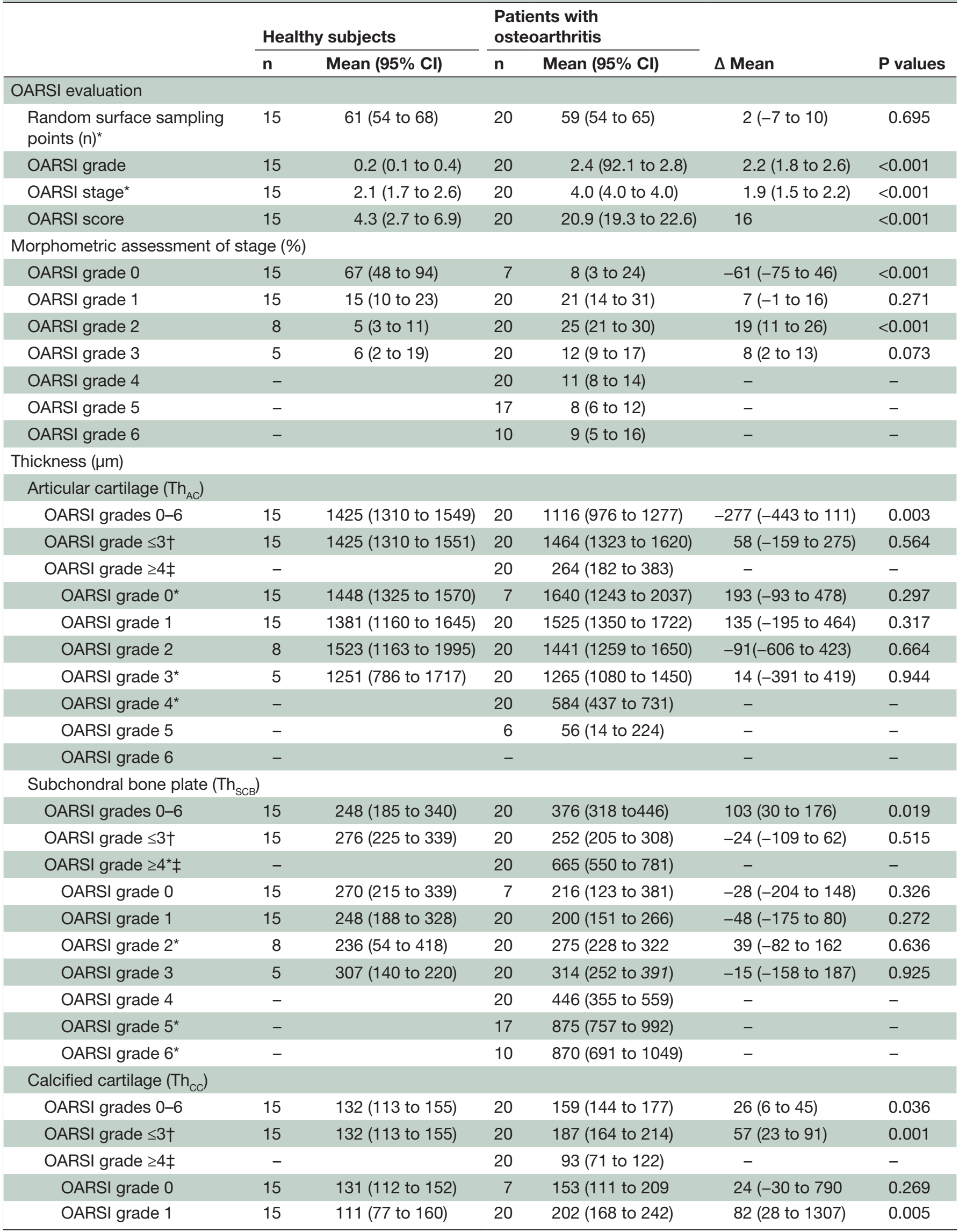




\begin{tabular}{|c|c|c|c|c|c|c|}
\hline & \multicolumn{2}{|c|}{ Healthy subjects } & \multicolumn{2}{|c|}{$\begin{array}{l}\text { Patients with } \\
\text { osteoarthritis }\end{array}$} & \multirow[b]{2}{*}{$\Delta$ Mean } & \multirow[b]{2}{*}{$P$ values } \\
\hline & $\mathbf{n}$ & Mean $(95 \% \mathrm{Cl})$ & $\mathbf{n}$ & Mean $(95 \% \mathrm{Cl})$ & & \\
\hline OARSI grade 2 & 8 & 186 (129 to 269$)$ & 20 & 174 (145 to 208$)$ & $-17(-113$ to 78 & 0.703 \\
\hline OARSI grade $3^{*}$ & 5 & 177 (137 to 219 ) & 20 & 183 (144 to 223$)$ & $6(-74$ to 85$)$ & 0.815 \\
\hline OARSI grade 4 & - & & 20 & 145 (125 to 168$)$ & - & - \\
\hline OARSI grade 5 & - & & 13 & 65 (37 to 113$)$ & - & - \\
\hline OARSI grade 6 & - & & 3 & 189 (1 to 583 ) & - & - \\
\hline
\end{tabular}

Data are presented as geometric mean and $(95 \% \mathrm{Cl})$. Statistical significance was found using Student's t-test. $\mathrm{P}<0.05$ was considered significant.

*Data that were normally distributed without being log transformed are presented as arithmetic mean $(95 \% \mathrm{Cl})$. TOARSI grade $\leq 3$ represents areas without loss of articular cartilage thickness.

¥OARSI grade $\geq 4$ represents areas with loss of articular cartilage thickness.

AC, articular cartilage;CC, calcified cartilage;OARSI, Osteoarthritis Research Society International;SCB, subchondral bone.

defined as the distance from the cement line until the first distal occurrence of bone marrow (figure 1C). ${ }^{28}$

\section{Thickness correction}

In order to fulfil the criteria of systematic uniform random sampling and vertical uniform random sections, the $7 \mathrm{~mm}$ thick halved parallel slices were sawed parallel to the VA. ${ }^{24}$ Hence, the measured thicknesses of the tissue structures were obtained perpendicular to the surface in the sawing plane, but not perpendicular to the curved three-dimensional joint surface. Therefore, each of the thickness measurements was corrected for oblique sectioning according to the location of the sawing plane. For full detail of the thickness correction, see online supplementary material.

\section{Coefficient of variance}

The variance of the applied method and the variance between femoral heads were evaluated using the coefficient of variance of the method $\left(\mathrm{CV}_{\text {Method }}\right)$ and the total coefficient of variance $\left(\mathrm{CV}_{\text {Total }}\right){ }^{29} \mathrm{CV}_{\text {Method }}$ ranged from 0.01 to 0.09 , while $\mathrm{CV}_{\text {Total }}$ ranged from 0.23 to 0.46 . Consequently, the variation observed could be attributed to the biological variation between the study subjects rather than the variance of the method. ${ }^{30}$

\section{Statistics}

Data were analysed using STATA V.12 (StataCorp, College Station, TX, USA). Normal distribution of the data was investigated with $\mathrm{Q}-\mathrm{Q}$ plots and histograms. Normally distributed data were presented as arithmetic mean $(95 \%$ CI). Data not being normally distributed were log-transformed, checked for normality and presented as geometric mean $(95 \% \mathrm{CI})$. The geometric mean difference between patients with $\mathrm{OA}$ and healthy subjects was found using bootstrapping with bias corrected and accelerated intervals to get $95 \%$ CI. Student's t-test was used to test for statistical significance between the patients with OA and the healthy subjects. The difference of thickness measurement was made for the complete femoral head (OARSI grades 0-6), for areas without loss of cartilage thickness (OARSI $\leq 3$ ) and for areas with loss of cartilage thickness (OARSI grade $\geq 4$ ). Student's t-test was also used for comparison between patients with $\mathrm{OA}$ and the healthy subjects regarding the thickness measurements for each individual OARSI grade. One-way analysis of variance (ANOVA) was used to investigate the difference of the thickness measurements according to OARSI grades in both patients with OA and healthy subjects. The post hoc Bonferroni test was used to identify specific differences in the thickness measurements between corresponding OARSI grades. The results were considered significant at $\mathrm{p}<0.05$.

\section{RESULTS}

In the patients with $\mathrm{OA}$, the hip X-ray showed a mean Kellgren-Lawrence grade ${ }^{31}$ of 3.9 (3.8-4). The patients with $\mathrm{OA}$ had a mean Western Ontario and McMaster Universities Arthritis Index (WOMAC) ${ }^{32}$ score for pain of 49.9 (39.4-60.3), for stiffness of 58.2 (45.2-71.2) and physical activity of 49.9 (33.2-47.1). Neither the WOMAC score nor the Kellgren-Lawrence grade could be obtained from the healthy subjects (table 1 ).

\section{OARSI grading, staging and scoring}

The mean number of random sampling points of the entire femoral head surface area did not differ between the patients with OA and the healthy subjects (table 2). The mean OARSI grade, stage and score for the entire femoral head were significantly higher for patients with OA compared with the healthy subjects (table 2).

Applying morphometric staging, the patients with OA had a mean of $18 \%(11 \%-21 \%)$ of the femoral head surface area with complete loss of AC (OARSI grade $\geq 5$ ). The femoral head of the healthy subjects, however, was covered entirely by AC of normal thickness (OARSI grade $\leq 3)$. In patients with $\mathrm{OA}, 72 \%(65 \%-80 \%)$ of the femoral head was covered by cartilage of normal thickness (OARSI grade $\leq 3$ )

\section{Thickness of AC}

The patients with OA had statistically significantly lower mean $\mathrm{Th}_{\mathrm{AC}}$ for the total joint surface area (OARSI grades 


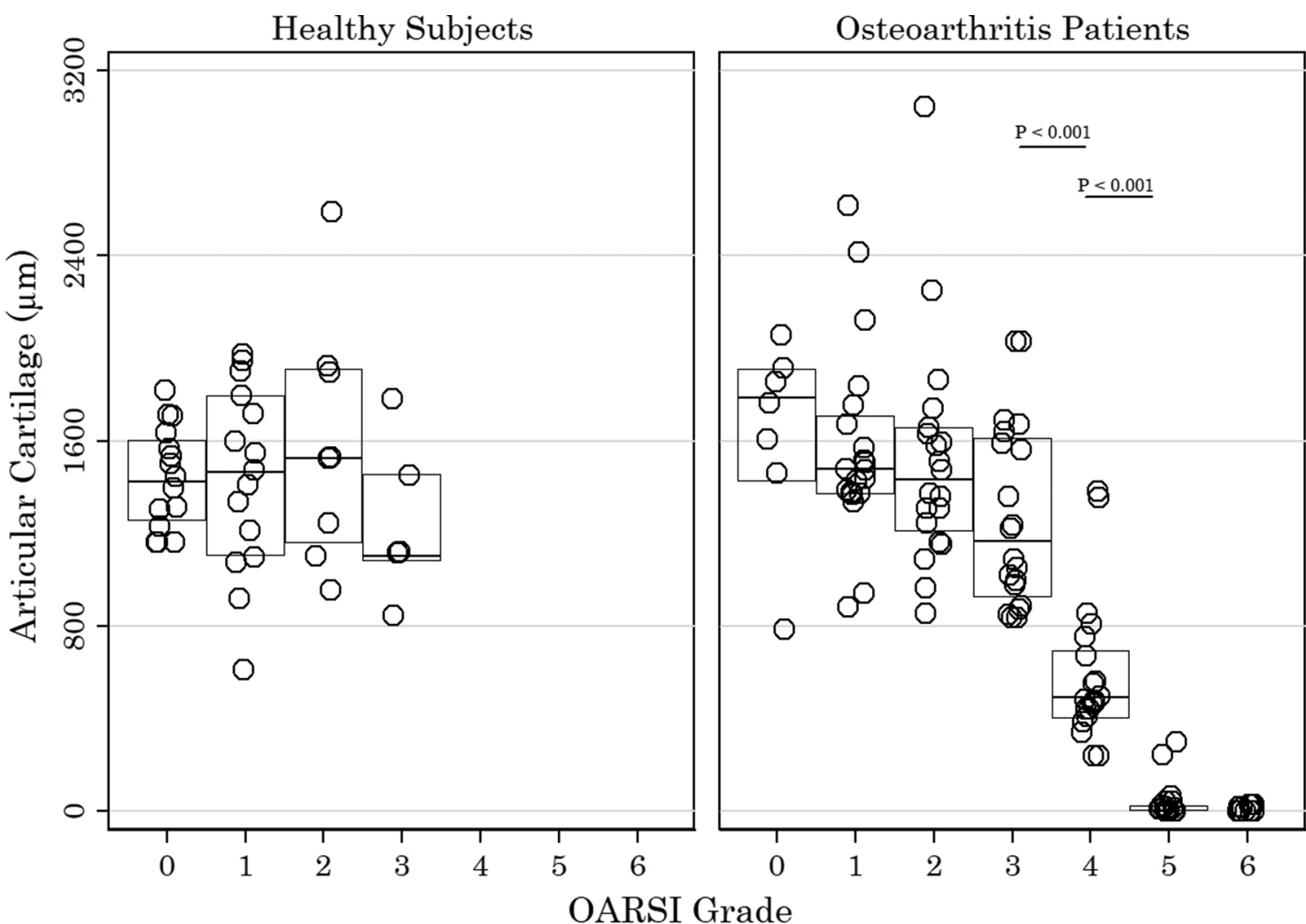

Figure 2 The thickness of the articular cartilage for healthy subjects and patients with osteoarthritis (OA). Each circle represents the articular cartilage thickness categorised according to the OARSI grades 0-6 in an individual. The horizontal line indicates the median while the IQR is represented by the boxes. One-way analysis of variance for $T_{A C}$ according to OARSI grade was significant for the patients with $\mathrm{OA}(F(5,87)=62.06, \mathrm{p}<0.001)$ but not for healthy subjects $(F(3,39)=0.82, \mathrm{p}=0.0491)$. The $p$ values indicated are the result of post hoc Bonferroni test. $\mathrm{P}<0.05$ was considered significant. OARSI, Osteoarthritis Research Society International.

0-6) compared with healthy subjects (table 2). There was no significant difference for $\mathrm{Th}_{\mathrm{AC}}$ between patients with $\mathrm{OA}$ and healthy subjects in areas without loss of cartilage thickness (OARSI grade $\leq 3$ ) (table 2). For patients with $\mathrm{OA}, \mathrm{Th}_{\mathrm{AC}}$ for areas with loss of cartilage thickness (OARSI grade $\geq 4$ ) was significantly thinner compared with $\mathrm{Th}_{\mathrm{AC}}$ for areas without loss of cartilage thickness (OARSI grade $\leq 3) \quad(p<0.001)$. According to individual OARSI grades, there was no significant difference in AC thickness between the patients with OA and healthy subjects (table 2).

One-way ANOVA for $\mathrm{Th}_{\mathrm{AC}}$ according to OARSI grade was significant for the patients with OA $(F(5,87)=62.06$, $\mathrm{p}<0.001)$ but not for healthy subjects $(F(3,39)=0.82$, $\mathrm{p}=0.491)$. The mean $\mathrm{Th}_{\mathrm{AC}}$ in patients with OA decreased significantly from OARSI grades 3-5 (figure 2).

\section{Thickness of SCB}

The patients with OA had statistically significantly higher mean $\mathrm{Th}_{\mathrm{SCB}}$ for the total joint surface area (OARSI grades $0-6)$ compared with healthy subjects (table 2 ). $\mathrm{The}_{\mathrm{SCB}}$ for areas without loss of cartilage thickness (OARSI grade $\leq 3$ ) was not significantly different for patients with OA compared with healthy subjects (table 2). According to individual OARSI grades, there was no significant difference in SCB thickness between the patients with OA and healthy subjects (table 2). For patients with $\mathrm{OA}, \mathrm{Th}_{\mathrm{SCB}}$ for areas with loss of cartilage thickness (OARSI grade $\geq 4$ ) was significantly thicker compared with $\mathrm{Th}_{\mathrm{SCB}}$ for areas without loss of cartilage thickness (OARSI grade $\leq 3)(\mathrm{p}<0.001)$. One-way ANOVA for $\mathrm{Th}_{\mathrm{SCB}}$ according to OARSI grade was significant for the patients with OA $(F(6,107)=23.23, \mathrm{p}<0.001)$ but not for healthy subjects $(F(3,39)=1.34, \mathrm{p}=0.274)$. The higher mean $\mathrm{Th}_{\mathrm{SCB}}$ of patients with OA was seen only in areas with total loss of AC, that is, denuded bone surface (OARSI grades 5-6) (figure 3).

\section{Thickness of CC}

The mean thickness of the CC for the total joint surface area (OARSI grades 0-6) was significantly higher for patients with OA compared with the healthy subjects (table 2). For areas without loss of cartilage thickness (OARSI grade $\leq 3$ ), this difference was even greater between the patients with $\mathrm{OA}$ and the healthy subjects (table 2). According to individual OARSI grades, there was significant difference in CC thickness between the patients with OA and healthy subjects at OARSI grade 1 (table 2). For patients with $\mathrm{OA}$, the $\mathrm{Th}_{\mathrm{CC}}$ in areas with loss of cartilage (OARSI grade $\geq 4$ ) was significantly thinner compared with $\mathrm{Th}_{\mathrm{CC}}$ for areas without loss of cartilage $($ OARSI grade $\leq 3)(\mathrm{p}=0.001)$. One-way ANOVA for $\mathrm{Th}_{\mathrm{CC}}$ 


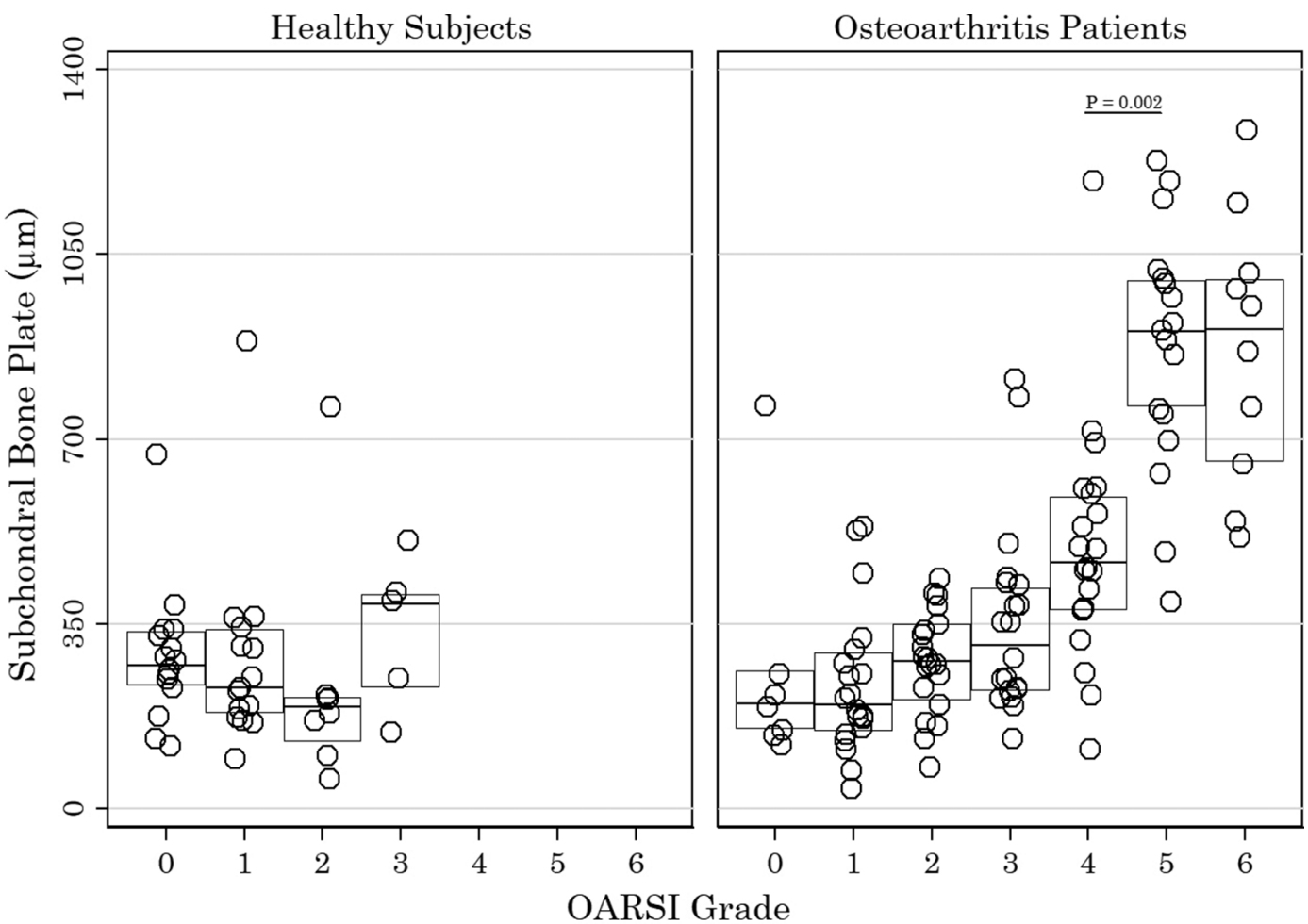

Figure 3 The thickness of the subchondral bone plate for healthy subjects and patients with osteoarthritis (OA). Each circle represents the subchondral bone plate thickness categorised according to the OARSI grades 0-6 in an individual. The horizontal line indicates the median while the IQR is represented by the boxes. One-way analysis of variance for Th $\mathrm{scB}_{\mathrm{s}}$ according to OARSI grade was significant for the patients with $O A(F(6,107)=23.23, p<0.001)$ but not for healthy subjects $(F(3,39)=1.34, \mathrm{p}=0.274)$. The $\mathrm{p}$ values indicated are the result of post hoc Bonferroni test. $\mathrm{P}<0.05$ was considered significant. OARSI, Osteoarthritis Research Society International.

according to OARSI grade was significant for the patients with OA $(F(6,96)=14.5, \mathrm{p}<0.001)$ but not for healthy subjects $(F(3,39)=2.61, \mathrm{p}=0.065)$. The mean $\mathrm{Th}_{\mathrm{CC}}$ in patients with OA decreased significantly from OARSI grade 4 to grade 6 (figure 4 ).

\section{DISCUSSION}

This study is the first to investigate the thickness of the different tissues in the bone-cartilage unit and their relation to cartilage damage using entire human femoral heads and design-based stereological sampling. The study confirmed that the CC was thicker in patients with OA compared with healthy subjects and that this was present at early stages without loss of AC. The thickening of the SCB plate, however, occurred only in areas where the AC was completely lost.

\section{Subchondral bone}

It is well known that $\mathrm{OA}$ is characterised by thickening of the SCB plate..$^{214}$ In the present study, however, we found that the thicker SCB plate in patients with OA only occurred in areas with denuded bone surface (OARSI grades 5-6) where the SCB plate was more than twofold thicker compared with areas with OARSI grade 4. This is in accordance with our previous study, which found that the SCB volume was higher in patients with OA compared with healthy subjects but only in areas with denuded bone surface. ${ }^{5}$ Therefore, severe bone thickening in areas with denuded bone surface is probably also a result of increased biomechanical load and a reactive increase in bone modelling in favour of bone formation by appositional growth. ${ }^{21}$ This is supported by studies showing increased bone turnover ${ }^{5}$ of hypomineralised $^{1433}$ and severely thickened SCB in OA. ${ }^{34}$

\section{Calcified cartilage}

The patients with OA had significantly thicker CC compared with the healthy subjects, which was observed in areas without loss of cartilage thickness (OARSI grade $\leq 3)$. A thicker CC in early OA indicates an early advancement of the CC into the AC and is suggestive of bone growth by endochondral ossification, ${ }^{8}$ which can lead to the replacement of the AC by bone. ${ }^{35}$

Only a few studies have measured the thickness of the CC in patients with OA. Revell et al showed no difference in human patellae between patients with OA and healthy subjects. ${ }^{33}$ The different outcome, by Revell et al, can be explained by location, smaller sample size, and that they sampled the central patella only, whereas we applied an unbiased random sampling procedure to measure the thickness of the entire femoral head joint surface, that is, including areas of all OARSI grades. Other studies have shown patients with OA to have a larger tidemark area in the patella compared with the healthy subjects ${ }^{36}$ 


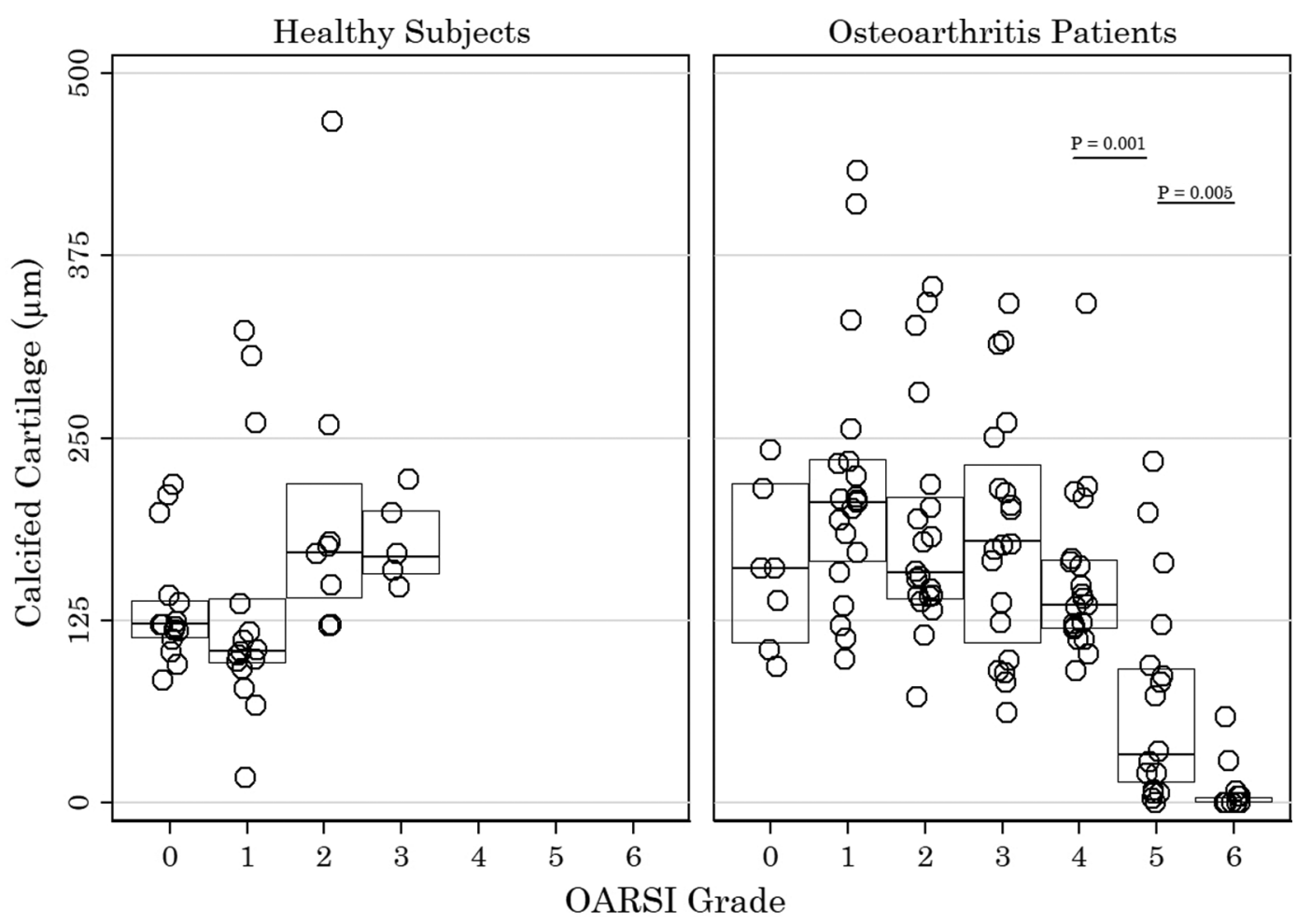

Figure 4 The thickness of the calcified cartilage for healthy subjects and patients with osteoarthritis (OA). Each circle represents the calcified cartilage thickness categorised according to the OARSI grades 0-6 in an individual. The horizontal line indicates the median while the IQR is represented by the boxes. One-way analysis of variance for Th $\mathrm{CC}_{\mathrm{C}}$ according to OARSI grade was significant for the patients with $\mathrm{OA}(F(6,96)=14.5, \mathrm{p}<0.001)$ but not for healthy subjects $(F(3,39)=2.61, \mathrm{p}=0.065)$. The $\mathrm{p}$ values indicated are the result of post hoc Bonferroni test. $\mathrm{P}<0.05$ was considered significant. OARSI, Osteoarthritis Research Society International.

and increased mineralisation of the $\mathrm{AC},{ }^{37}$ suggesting an increased metabolic activity of the $\mathrm{CC}$ in $\mathrm{OA}$, which is in line with our findings in the present study.

A study by Deng et al found that the CC thickness increased progressively from OARSI grade 0 to grade 4 with the exception of OARSI grade $2 .^{38}$ The authors interpreted the thinning of the CC at OARSI grade 2 as a result of early SCB plate growth evidenced by the increased irregularity of the cement line. Under normal conditions, cement line irregularity is influenced by SCB remodelling, and vascular invasion, ${ }^{39}$ which is increased in OA. ${ }^{520}$ In the present study, the CG was thicker in patients with OA compared with healthy subjects, and this was present also in low to moderate, that is, OARSI grade $\leq 3$. However, the $\mathrm{Th}_{\mathrm{CC}}$ declined progressively in moderate to severe OA from OARSI grade 4 to grade 6 , subsequently to AC thinning and at the same stage as SCB plate thickening. Thus, our findings point towards CC thickening as a precursor for SCB plate thickening and suggest endochondral ossification and reactivation of the secondary ossification centre. ${ }^{8}$ This is supported by studies showing an irregularity of the cement line ${ }^{83}$ and vascularisation across the $\mathrm{CC}$ allowing crosstalk between the SCB plate and the AC. 363940

\section{Articular cartilage}

As expected, the overall $\mathrm{AC}$ was thinner in patients with hip OA compared with healthy subjects. Reduced cartilage thickness in patients with OA was only observed in areas with OARSI grade $\geq 4$. Our estimate of the $\mathrm{Th}_{\mathrm{AC}}$ was therefore in accordance with the OARSI scoring method. ${ }^{22}$ Joint surface with loss of cartilage thickness (OARSI grade $\geq 4$ ), however, only accounted for approximately one-fifth of the femoral head surface area in patients with OA. Radiological OA severity is determined by bone sclerosis, osteophyte formation and joint space narrowing, ${ }^{31}$ but as we have demonstrated, severe thickening of the SCB appears to be a marker of focal highgrade osteoarthritic lesions occurring together with complete focal loss of AC. This may partly explain the difficulties in using subchondral bone sclerosis and joint space narrowing from plain radiographs as a marker of OA disease progression.

\section{Strength and limitations}

The present study is the first to fully apply the histopathology assessment methodology proposed by Pritzker $e t a l^{22}$ By the use of this technique, the entire femoral head was studied as a complete architectural object. We applied morphometric assessment of grade and stage, by combining the stereological design of systematic uniform random sampling, and vertical uniform random sections of the entire joint surface area with histological OARSI grading in the human hip OA. This method has the advantage over the OARSI score that the assessment is not biased towards the most advanced OA pathology 
observed. ${ }^{22}$ The morphometric assessment of grade and stage gives unbiased semiquantitative scores but is time consuming and requires specific expertise.

As the current study is a cross-sectional study, we were not able to directly investigate the time course of OA progression. However, if patients with $\mathrm{OA}$ represent subjects with a predisposition for developing OA, then regions with low-grade osteoarthritic lesions may be considered to represent early-stage OA.

In the present work, correction for tissue shrinkage during the histology processing was not performed. It has previously been demonstrated that cartilage and bone specimens from porcine hips embedded in methylmethacrylate show shrinkage of less than $2 \%$, which can be regarded as negligible. ${ }^{41}$

\section{CONCLUSIONS}

In the present cross-sectional study of human femoral heads from patients with $\mathrm{OA}$ and healthy subjects, we showed that the OA pathology of the CC and the SCB bone plate does not indicate progressively developing disease progression. The $\mathrm{CC}$ was thicker in $\mathrm{OA}$, and this was present in areas without loss of AC thickness, which suggests that endochondral ossification is an early phenomenon of OA. In moderate osteoarthritic lesions, a thicker SCB plate and thinner AC further support the hypothesis that the CC may serve as a growth plate for endochondral ossification in human OA. However, significantly thickening of the SCB plate was a late-stage phenomenon of denuded bone surface only, suggesting that appositional bone growth may occur as a consequence of changed biomechanics.

\section{Author affiliations \\ ${ }^{1}$ Department of Rheumatology, Aarhus University Hospital, Aarhus, Denmark ${ }^{2}$ Department of Biomedicine - Anatomy, Aarhus University, Aarhus, Denmark ${ }^{3}$ Department of Clinical Medicine,Core Centre for Molecular Morphology, Section for Stereology and Microscopy, Centre for Stochastic Geometry and Advanced Bioimaging, Aarhus University, Aarhus, Denmark \\ ${ }^{4}$ Institute of Forensic Medicine, Aarhus University, Aarhus, Denmark \\ ${ }^{5}$ Orthopaedic Surgery Research Unit, Aalborg Hospital - Aarhus University Hospital, Aalborg, Denmark \\ ${ }^{6}$ Department of Clinical Medicine, Aarhus University, Aarhus, Denmark}

Acknowledgements The authors are grateful for the excellent technical assistance of Rita Ullerup and Jette Barlach.

Contributors All authors were involved in drafting the article or revising it critically for important intellectual content, and all authors approved the final version to be published. Study conception and design: LBH, JST, JRN and EMH. Acquisition of tissue: LBH, LWTB, MBL, TBL and EMH. Analysis and interpretation of data: RKJ, LBH, JST, JRN, AWN and EMH.

Funding The study has been financially supported by the Danish Council for Independent Research (Medical Science), Danish Rheumatism Association, HN Family Foundation, Kathrine and Vigo Skovgaards Foundation, Henny and Helge Holgersens Grant, Aase and Ejnar Danielsen's Foundation, Dagmar Marshall's Foundation, Managing Director Ib Henriksen's Foundation, Managing Director Jacob Madsen and wife Olga Madsen's Foundation, the Beckett Foundation, the Gangsted Foundation, Gerda and Aage Haensch's Foundation, the Hørslev Foundation, the Illum Foundation and the Valdemar Foersom and wife Thyra Foersom's Foundation. The Centre for Stochastic Geometry and Advanced Bioimaging is supported by Villum Foundation.

Competing interests EMH reports personal fees from MSD, Pfizer, UCB and Sobi; grants from Roche and Novartis, outside the submitted work. All other authors have no conflicts of interest to declare.
Patient consent Obtained.

Ethics approval The Central Denmark Region Committees on Health Research Ethics (J number 10776).

Provenance and peer review Not commissioned; externally peer reviewed. Data sharing statement No additional data are available.

Open access This is an open access article distributed in accordance with the Creative Commons Attribution Non Commercial (CC BY 4.0) license, which permits others to distribute, remix, adapt, build upon this work non-commercially, and license their derivative works on different terms, provided the original work is properly cited and the use is non-commercial. See: http://creativecommons.org/ licenses/by/4.0

\section{REFERENCES}

1. Daghestani HN, Kraus VB. Inflammatory biomarkers in osteoarthritis. Osteoarthritis Cartilage 2015;23:1890-6.

2. Burr DB, Gallant MA. Bone remodelling in osteoarthritis. Nat Rev Rheumatol 2012;8:665-73.

3. Heinegård $D$, Saxne $T$. The role of the cartilage matrix in osteoarthritis. Nat Rev Rheumatol 2011;7:50-6.

4. Sellam J, Berenbaum $F$. The role of synovitis in pathophysiology and clinical symptoms of osteoarthritis. Nat Rev Rheumatol 2010;6:625-35.

5. Klose-Jensen R, Hartlev LB, Boel LWT, et al. Subchondral bone turnover, but not bone volume, is increased in early stage osteoarthritic lesions in the human hip joint. Osteoarthritis Cartilage 2015;23:2167-73.

6. Park DY, Jin LH, Min BH, et al. Subchondral bone scan uptake correlates with articular cartilage degeneration in osteoarthritic knees. Int J Rheum Dis 2017;20:1393-402.

7. Lories RJ, Luyten FP. The bone-cartilage unit in osteoarthritis. Nat Rev Rheumatol 2011;7:43-9.

8. Burr DB, Radin EL. Microfractures and microcracks in subchondral bone: are they relevant to osteoarthrosis? Rheum Dis Clin North Am 2003;29:675-85

9. Brandt KD, Radin EL, Dieppe PA, et al. Yet more evidence that osteoarthritis is not a cartilage disease. Ann Rheum Dis 2006;65:1261-4.

10. Saito T, Fukai A, Mabuchi A, et al. Transcriptional regulation of endochondral ossification by HIF-2alpha during skeletal growth and osteoarthritis development. Nat Med 2010;16:678-86.

11. Aspden RM, Hukins DW. Collagen organization in articular cartilage, determined by X-ray diffraction, and its relationship to tissue function. Proc R Soc Lond B Biol Sci 1981;212:299-304.

12. Li B, Aspden RM. Composition and mechanical properties of cancellous bone from the femoral head of patients with osteoporosis or osteoarthritis. J Bone Miner Res 1997;12:641-51.

13. Dedrick DK, Goldstein SA, Brandt KD, et al. A longitudinal study of subchondral plate and trabecular bone in cruciate-deficient dogs with osteoarthritis followed up for 54 months. Arthritis Rheum 1993;36:1460-7.

14. Grynpas MD, Alpert B, Katz I, et al. Subchondral bone in osteoarthritis. Calcif Tissue Int 1991;49:20-6.

15. Bobinac D, Spanjol J, Zoricic S, et al. Changes in articular cartilage and subchondral bone histomorphometry in osteoarthritic knee joints in humans. Bone 2003;32:284-90.

16. Altman R, Alarcón G, Appelrouth D, et al. The American College of Rheumatology criteria for the classification and reporting of osteoarthritis of the hip. Arthritis Rheum 1991;34:505-14.

17. Altman RD, Gold GE. Atlas of individual radiographic features in osteoarthritis, revised. Osteoarthritis Cartilage 2007;15:A1-A56.

18. Jeffery AK. Osteophytes and the osteoarthritic femoral head. J Bone Joint Surg Br 1975;57:314-24.

19. Burr DB, Schaffler MB. The involvement of subchondral mineralized tissues in osteoarthrosis: quantitative microscopic evidence. Microsc Res Tech 1997;37:343-57.

20. Burr DB. Anatomy and physiology of the mineralized tissues: role in the pathogenesis of osteoarthrosis. Osteoarthritis Cartilage 2004; $12: 20-30$.

21. Cox LG, van Rietbergen B, van Donkelaar CC, et al. Bone structural changes in osteoarthritis as a result of mechanoregulated bone adaptation: a modeling approach. Osteoarthritis Cartilage 2011:19:676-82.

22. Pritzker KP, Gay S, Jimenez SA, et al. Osteoarthritis cartilage histopathology: grading and staging. Osteoarthritis Cartilage 2006;14:13-29. 
23. Hartlev LB, Nyengaard JR, Thomsen JS, et al. Application of designbased stereology for estimation of absolute volume and surface area of the articular and calcified cartilage compartments of undecalcified human femoral heads. J Microsc 2013;251:133-43.

24. Baddeley AJ, Gundersen HJ, Cruz-Orive LM. Estimation of surface area from vertical sections. J Microsc 1986;142(Pt 3):259-76.

25. Boyce RW, Dorph-Petersen KA, Lyck L, et al. Design-based stereology: introduction to basic concepts and practical approaches for estimation of cell number. Toxicol Pathol 2010;38:1011-25.

26. Jensen EB, Gundersen HJ, Østerby R. Determination of membrane thickness distribution from orthogonal intercepts. J Microsc 1979;115:19-33.

27. Madry H, van Dijk CN, Mueller-Gerbl M. The basic science of the subchondral bone. Knee Surg Sports Traumatol Arthrosc 2010;18:419-33.

28. de Bri E, Reinholt FP, Svensson O. Primary osteoarthrosis in guinea pigs: a stereological study. J Orthop Res 1995;13:769-76.

29. Gundersen HJ, Bendtsen TF, Korbo L, et al. Some new, simple and efficient stereological methods and their use in pathological research and diagnosis. APMIS 1988;96:379-94.

30. Nyengaard JR. Stereologic methods and their application in kidney research. J Am Soc Nephrol 1999;10:1100-23.

31. Kellgren JH, Lawrence JS. Radiological assessment of osteoarthrosis. Ann Rheum Dis 1957;16:494-502.

32. Bellamy N, Buchanan WW. A preliminary evaluation of the dimensionality and clinical importance of pain and disability in osteoarthritis of the hip and knee. Clin Rheumatol 1986;5:231-41.
33. Revell PA, Pirie C, Amir G, et al. Metabolic activity in the calcified zone of cartilage: observations on tetracycline labelled articular cartilage in human osteoarthritic hips. Rheumatol Int 1990;10:143-7.

34. Cox LG, van Donkelaar CC, van Rietbergen B, et al. Decreased bone tissue mineralization can partly explain subchondral sclerosis observed in osteoarthritis. Bone 2012;50:1152-61.

35. Hosaka Y, Saito T, Sugita S, et al. Notch signaling in chondrocytes modulates endochondral ossification and osteoarthritis development. Proc Natl Acad Sci USA 2013;110:1875-80.

36. Bonde HV, Talman ML, Kofoed $\mathrm{H}$. The area of the tidemark in osteoarthritis--a three-dimensional stereological study in 21 patients. APMIS 2005;113:349-52.

37. Fuerst M, Niggemeyer O, Lammers L, et al. Articular cartilage mineralization in osteoarthritis of the hip. BMC Musculoskelet Disord 2009;10:166.

38. Deng B, Wang F, Yin L, et al. Quantitative study on morphology of calcified cartilage zone in OARSI 0 4 cartilage from osteoarthritic knees. Curr Res Trans/ Med 2016;64:149-54.

39. Lane LB, Villacin A, Bullough PG. The vascularity and remodelling of subchondrial bone and calcified cartilage in adult human femoral and humeral heads. An age- and stress-related phenomenon. $J$ Bone Joint Surg Br 1977;59:272-8.

40. Pan J, Wang B, Li W, et al. Elevated cross-talk between subchondral bone and cartilage in osteoarthritic joints. Bone 2012;51:212-7.

41. Ferguson SJ, Bryant JT, Ito K. Three-dimensional computational reconstruction of mixed anatomical tissues following histological preparation. Med Eng Phys 1999;21:111-7. 